\title{
Evaluation of Ultrasonographic Findings in Dengue Fever Cases during an Outbreak at a Tertiary Care Hospital of South India
}

\author{
Gayatri Manam ${ }^{1}$, Ravi M Godavarthi' ${ }^{2}$, Ramakrishna Baru ${ }^{3}$, Sunitha ${ }^{4}$, Gowtham Surya Duddu ${ }^{5}$ \\ ${ }^{1}$ Assistant Professor, Department of Radiology, ${ }^{2}$ Associate Professor, Department of Radiology, ${ }^{3}$ Professor. Department \\ of Radiology, ${ }^{4}$ Professor Department of Radiology, ${ }^{5}$ Resident, Department of Radiology, Narayana Medical College \\ Chinthareddypalem, Nellore, Andhra Pradesh, India
}

Corresponding author: Dr. Ravi M Godavarthi, Associate Professor, Department of Radiology, arayana Medical College Chinthareddypalem, Nellore, Andhra Pradesh, India

DOI: $10.21276 /$ ijcmsr.2018.3.2.26

How to cite this article: Gayatri Manam, Ravi M Godavarthi, Ramakrishna Baru, Sunitha, Gowtham Surya Duddu. Evaluation of ultrasonographic findings in dengue fever cases during an outbreak at a tertiary care hospital of South India. International Journal of Contemporary Medicine Surgery and Radiology. 2018;3(2):B106B110.

\section{A B S T R A C T}

Introduction: Dengue virus infections are a significant threat to human populations particularly in the tropical and subtropical regions. This is mainly transmitted by Aedes aegypti mosquito from humans to humans. The main pathological mechanisms associated include thrombocytopenia, hemorrhagic diathesis with hemoconcentration. Our present study was aimed to study the Ultrasonographic features of chest and abdomen cases of dengue fever of all types during an outbreak prospectively.

Material and methods: A prospective study for a period of one year was conducted during an outbreak after ethical committee approval. All the cases of dengue fever serologically diagnosed were evaluated for ultra sonogram abdomen and chest and findings were recorded. Platelet counts were estimated in all the cases and compared with findings recorded on ultra sonogram. Qualitative variables such as presence of various ultrasound findings were expressed as percentages and their association with platelet counts or ages were assessed through chi-square test and a $p$ value $<0.05$ was considered significant.

Results: Males were predominant in our study (59.26\%) with male: female ratio of 1.45:1. Common age group of the study was 11-29 years. Gall ball thickness was the most common USG finding in our study (65.08\%) followed by pleural effusion (49.21\%). Gallbladder wall thickening was the most common finding in Group -2 and group-3 with around $71 \%$. Ascites with hepatomegaly was the commonest in group -1 cases, Thickness of gall bladder wall with pleural effusion in Group -2 cases, spleenomegaly and hepatomegaly in Group -3 and Group- 4 cases.

Discussion: In our study, thickness of the gall bladder wall was the most sensitive finding and pleural effusion was the most specific finding. USG is a relevant and important tool for early detection of plasma leakage signs and helps in follow up of patients who have a risk to progress to DHF. GB thickening with or without polyserosistis in a febrile patient should suggest the possibility of Dengue fever during an epidemic outbreak.

Key words: Dengue fever, Gall bladder wall thickness, Ascites, Hepatomegaly, Spleenomegaly.

\section{INTRODUCTION}

Dengue virus infections are a significant threat to human populations particularly in the tropical and subtropical regions. Dengue fever is known for more than centuries in countries of South East Asia and Western Pacific regions. Up to 50-100 million infections are estimated to occur annually in all over 100 endemic countries putting half of world population at risk. This disease is mainly prevalent in India, Indonesia, Thailand, Myanmar, Bangladesh and Srilanka. ${ }^{1,2}$ Dengue virus which comprises four distinct serological types is an Arbovirus belonging to Falviviridae family. This is mainly transmitted by Aedes aegypti mosquito from humans to humans. Dengue virus infections are once restricted to urban areas but they are now present in rural areas also. Many studies reported changing spatial patterns in dengue transmission. ${ }^{3}$ Infection with any of the four serotypes is associated with development of broad spectrum of illness ranging from asymptomatic to severe life threatening features. These features can be a mild dengue fever to severe Dengue Hemorrhagic fever and shock. Clinically patient presents with severe headache, high fever with chills, retro-orbital pain and severe backache. The main pathological mechanisms associated include thrombocytopenia, hemorrhagic diathesis with hemoconcentration. The diagnosis of these cases mainly depend on the serological demonstration of virus Non specific antigen 1 (NS1) and demonstration of antibodies Ig $\mathrm{G}$ and $\mathrm{Ig} \mathrm{M}$ against the viral antigen. Demonstration of antigen with reliability can be performed by rapid test from 
$2^{\text {nd }}$ day of the disease whereas demonstration of antibodies requires 5-7 days of time. Early diagnosis and suspicion in dengue fever might help in reducing the complications and in management of cases of dengue hemorrhagic fever or shock. ${ }^{4}$

Our present study was aimed to study the Ultrasonographic features of chest and abdomen cases of dengue fever of all types during an outbreak prospectively. Our study also correlated the findings with platelet count in the fever and their incidence with different age group of our study and to predict whether ultrasound helps in assessing the severity of the disease.

\section{MATERIAL AND METHODS}

A prospective study was conducted at a tertiary care hospital by the department of Radiology for a period of one year from January 2016 to December 2017. The study was approved by the institutional ethical committee and all the necessary protocols were followed during the study period. Protocol of the study was explained for all the cases who participated and written informed consent was obtained. Ultrasound examination was conducted on all the cases which were serologically diagnosed as Dengue based on the clinical signs and presentations. Radiological investigations included ultrasound chest, abdomen and computed tomography of the brain or chest radiography or a combination of above investigations.

Serological confirmation of the cases were done by department of Microbiology in the central laboratory by using Dengue card test by qualitative detection of NS1 antigen and Ig G and $\operatorname{IgM}$ antibodies to dengue virus antigen.

\begin{tabular}{|l|c|}
\hline USG features & Number (\%) \\
\hline Thickening of Gall bladder & $246(65.08 \%)$ \\
\hline Pleural effusion (Total) & $186(49.21 \%)$ \\
\hline Bilateral & $102(54.84 \%)$ \\
\hline Isolated right & $16(8.60 \%)$ \\
\hline Isolated left & $237(62.70 \%)$ \\
\hline Ascites & $108(28.57 \%)$ \\
\hline Hepatomegaly & $114(30.16 \%)$ \\
\hline Spleenomegaly & $82(21.69 \%)$ \\
\hline Normal & 378 \\
\hline Total number of cases & fever \\
\hline Table-1: Incidence of different sonographic findings in Dengue \\
\hline \multicolumn{2}{|c|}{} \\
\hline
\end{tabular}

Ultrasound examination was performed by using Philips Clear View 350 machine using linear (Hi frequency -7 to $12 \mathrm{MHz}$ ) and curvilinear (Low frequency-2-5 MHz) probes. Scanning was performed by a senior most radiologist and the findings were noted. Cases were scanned in supine and or sitting posture. Pleural spaces were evaluated by inter-coastal approach in sitting position. Ultrasound abdominal scan was performed after 4-6 hours of fasting to visualize the gall bladder distention preferably in early morning. Subjects with GB wall thickness $>3 \mathrm{~mm}$ on ultrasound were considered positive for gall bladder edema. Liver measuring more than $15 \mathrm{~cm}$ was taken as hepatomegaly and spleen measuring more than $12 \mathrm{~cm}$ was taken as spleenomegaly.

The demographic factors of all the cases including age, sex, and duration of fever, clinical signs and symptoms, laboratory parameters like $\mathrm{Hb} \%$, platelet count were noted and tabulated.

\section{STATISTICAL ANALYSIS}

All the data was entered in a spread Microsoft excel sheet and data was entered and corrected. The corrected data was analyzed and evaluated using SPPS software version 20 for Windows 10. Qualitative variables such as presence of various ultrasound findings were expressed as percentages and their association with platelet counts or ages were assessed through chi-square test and a $\mathrm{p}$ value $<0.05$ was considered significant.

\section{RESULTS}

In the present prospective study, a total of 378 cases were enrolled in the study. $59.26 \%$ of them were males (224/378) and $40.74 \%$ were females $(154 / 378)$. Male to female ratio in the study was $1.45: 1$. Mean age of the study population was $34.14 \pm 2.5$ with range of 6 years to 64 years. Mean age of the male in the study was $34.28 \pm 3.6$ years and female was $31.04 \pm 2.8$ years. All the cases in the study were categorized into four groups 1 to 4 . Group -1 (0-9 years) included 68 cases (17.99\%), Group-II (11-29 years) 124 cases (32.8\%), Group-III (30-49 years) 108 cases (28.57\%) and GroupIV (>49 years) 78 cases (20.63\%), Dengue fever was most common between ages of 11-29 years in our study.

Table -1 summarizes the findings of ultra sonogram among the cases in the study. Thickening of the gall bladder wall was the commonest finding (65.08\%) followed in order by pleural effusion (49.21\%), Ascites (62.7\%), spleenomegaly (30.16\%) and hepatomegaly (28.57\%). 82 cases (21.69\%) had no

\begin{tabular}{|c|c|c|c|c|c|}
\hline \multirow[t]{2}{*}{ USG features } & \multicolumn{4}{|c|}{ Number of Patients (\% of Total) } & \multirow{2}{*}{ p Value } \\
\hline & $\begin{array}{c}\text { Group } 1 \\
\text { (0-9 years) }\end{array}$ & $\begin{array}{c}\text { Group 2 } \\
(11-29) \text { years }\end{array}$ & $\begin{array}{c}\text { Group } 3 \\
(30-49) \text { years }\end{array}$ & $\begin{array}{c}\text { Group } 4 \\
>49 \text { years }\end{array}$ & \\
\hline Thickening of Gall bladder & $34(50 \%)$ & $89(71.77 \%)$ & 76 (70.37\%) & $47(60.26 \%)$ & 1.21 \\
\hline Pleural effusion & $23(33.82 \%)$ & $65(52.42 \%)$ & $43(39.81 \%)$ & $55(70.51 \%)$ & 0.84 \\
\hline Ascites & $32(47.06 \%)$ & $71(57.26 \%)$ & 76 (70.37\%) & $58(74.36 \%)$ & 1.01 \\
\hline Hepatomegaly & $26(38.24 \%)$ & $25(20.16 \%)$ & $25(23.15 \%)$ & $32(41.03 \%)$ & 0.75 \\
\hline Spleenomegaly & $18(26.47 \%)$ & $35(28.23 \%)$ & $31(28.7 \%)$ & $30(38.46 \%)$ & 0.89 \\
\hline Normal & $16(23.53 \%)$ & $17(13.71 \%)$ & $28(25.93 \%)$ & $21(26.92 \%)$ & 1.2 \\
\hline Total number of cases & $68(17.99 \%)$ & $124(32.8 \%)$ & $108(28.57 \%)$ & $78(20.63 \%)$ & \\
\hline
\end{tabular}




\begin{tabular}{|c|c|c|c|c|c|}
\hline \multirow[t]{2}{*}{ USG features } & \multicolumn{4}{|c|}{ Group } & \multirow[t]{2}{*}{ Total } \\
\hline & $\begin{array}{c}0-9 \\
\text { years }\end{array}$ & $\begin{array}{l}11-29 \\
\text { years }\end{array}$ & $\begin{array}{l}30-49 \\
\text { years }\end{array}$ & $\begin{array}{c}>49 \\
\text { years }\end{array}$ & \\
\hline GBT + ASC & 6 & 12 & 14 & 11 & 43 \\
\hline $\mathrm{GBT}+\mathrm{ASC}+\mathrm{PE}$ & 8 & 14 & 11 & 8 & 41 \\
\hline $\mathrm{GBT}+\mathrm{ASC}+\mathrm{PE}+\mathrm{SPM}$ & 5 & 21 & 14 & 14 & 54 \\
\hline $\mathrm{GBT}+\mathrm{ASC}+\mathrm{PE}+\mathrm{SPM}+\mathrm{HPM}$ & 3 & 14 & 13 & 10 & 40 \\
\hline $\mathrm{GBT}+\mathrm{ASC}+\mathrm{PE}+\mathrm{HPM}$ & 4 & 13 & 10 & 6 & 33 \\
\hline SPM+HPM & 8 & 20 & 21 & 19 & 68 \\
\hline $\mathrm{ASC}+\mathrm{PE}$ & 4 & 14 & 17 & 10 & 45 \\
\hline $\mathrm{ASC}+\mathrm{SPM}$ & 1 & 21 & 19 & 4 & 45 \\
\hline $\mathrm{GBT}+\mathrm{PE}$ & 10 & 24 & 20 & 15 & 69 \\
\hline GBT+ HPM & 8 & 11 & 8 & 4 & 31 \\
\hline $\mathrm{ASC}+\mathrm{HPM}$ & 12 & 14 & 10 & 9 & 45 \\
\hline
\end{tabular}

\begin{tabular}{|c|c|c|c|c|c|}
\hline \multirow[t]{2}{*}{ USG Changes } & \multicolumn{4}{|c|}{ Platelet count in cases of study } & \multirow[t]{2}{*}{$\mathrm{p}$ value } \\
\hline & $<40,000$ & $40,000-80,000$ & $81,000-1,50,000$ & $>1,50,000$ & \\
\hline Thickening of Gall bladder & $105(96.33 \%)$ & $54(77.14 \%)$ & $74(83.15 \%)$ & $13(46.43 \%)$ & 0.001 \\
\hline Pleural effusion & $84(77.06 \%)$ & $34(48.57 \%)$ & $54(60.67 \%)$ & $14(50 \%)$ & 0.01 \\
\hline Ascites & 98 (89.91\%) & $58(82.86 \%)$ & $63(70.79 \%)$ & $18(64.29 \%)$ & 0.01 \\
\hline Hepatomegaly & $50(45.87 \%)$ & $24(34.29 \%)$ & $24(26.97 \%)$ & $10(35.71 \%)$ & 0.22 \\
\hline Spleenomegaly & $54(49.54 \%)$ & $28(40 \%)$ & $20(22.47 \%)$ & $12(42.86 \%)$ & 0.48 \\
\hline Normal & 0 & $18(25.71 \%)$ & $39(43.82 \%)$ & $25(89.29 \%)$ & \\
\hline Total number of cases & 109 & 70 & 89 & 28 & \\
\hline
\end{tabular}

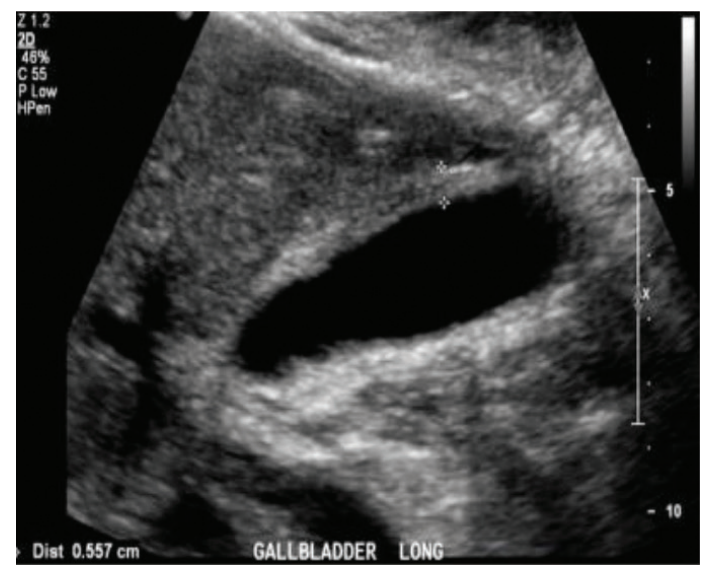

Image-1: Oedematous gall bladder wall in a case of 35 years old male.

abnormal findings on ultra sonogram of chest and abdomen. Among the cases of pleural effusion, 102 cases (54.84\%) had bilateral effusion, 68 cases $(36.56 \%)$ had isolated right sided effusion and 16 cases (8.6\%) had isolated left sided effusion. Gallbladder wall thickening was the most common finding in Group -2 and group-3 with around 71\%.Pleural effusion and Ascites was most common in group- 4 with $70.51 \%$ and $74.36 \%$. Hepatomegaly and spleenomegaly was common in Group - 4 cases with $41.03 \%$ and $38.46 \%$ of cases. Among the cases of Group - 1, gall bladder wall thickening was the commonest (50\%) and spleenomegaly was the least (26.47\%). In cases of Group -2, thickening of gall bladder was most common finding (71.77\%) and hepatomegaly was the least

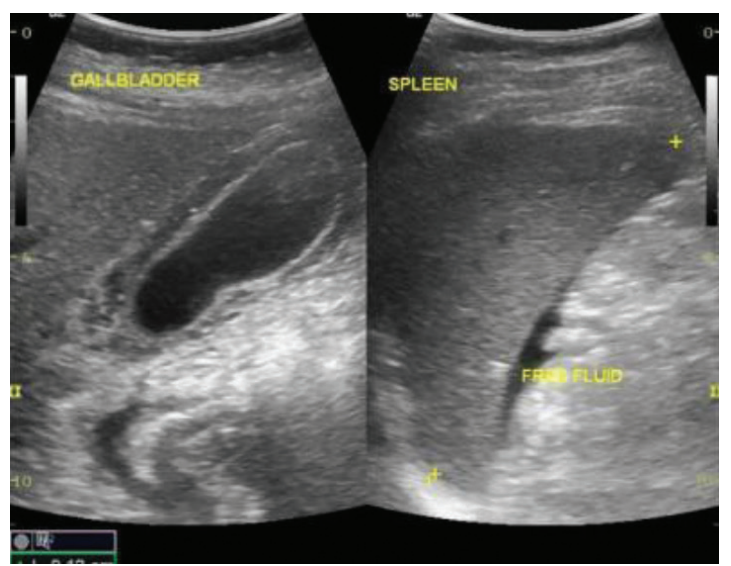

Image-2: Spleenomegaly and Gall bladder wall thickening in a case of DHF.

(20.16\%), In cases of Group - 3, gall bladder wall thickening and ascites were almost equally reported ( $70.37 \%$ ) and hepatomegaly was the least (23.15\%). In group -4 cases, ascites was the most common finding (74.36\%) followed by pleural effusion (70.51\%) and least was spleenomegaly with $38.46 \%$. [Table-2]

Table-3 summarizes the various combinations of ultra sound findings among the cases in our study. The commonest combination was Gallbladder thickness with pleural effusion and other was a combination of spleenomegaly and hepatomegaly. Ascites with hepatomegaly was the commonest in group -1 cases, Thickness of gall bladder wall with pleural effusion in Group -2 cases, spleenomegaly and 
hepatomegaly in Group - 3 and Group- 4 cases (image 1,2). In our study we correlated the Ultrasonographic findings with platelet count in all the cases of the study. Thickness of the gallbladder wall was most common in cases with platelet counts $<40,000$ and $81000-1,50,000$. Ascites was the most common feature observed than GB wall thickening in cases with platelet counts in range of $41,000-80,000$. Spleenomegaly and hepatomegaly were most commonly seen in cases with platelet counts $<40000$. In 82 cases of our study, no abnormal findings were observed in CT and ultrasound abdomen and chest even they were found serologically positive for dengue. Among the 82 cases with normal findings, maximum (39 cases) were with platelet counts between 81,000-1,50,000. A statistically significant association was observed with reduced platelet count $(<40,000)$ and thickness of GB wall, pleural effusion and ascites in all the cases of our study. [Table-4]

\section{DISCUSSION}

Dengue is considered as one of the most rapidly spreading mosquito borne disease throughout the world. Annually the incidence of Dengue cases is in a rise and there is a drastic shift from rural to urban population. In India the incidence of Dengue cases have increased drastically, due to rapid urbanization, lack of control measures for mosquitoes and poor water management during rainfalls. Epidemiologically dengue cases are more spread in areas with improper sanitary practices, lack of awareness of the disease, and changing urbanization in places with mass construction. A history of recent outbreaks in India documented changing clinical presentations from classical type of fever with high grade fever and chills, retro orbital pain, body pains to Atypical presentations like severe myalgia, low grade fever and less number of cases with classical backache. ${ }^{6}$

Dengue virus is a DNA arbovirus with four different serotypes DEN-1, 2, 3 and 4. Each serotype presents with similar pattern of clinical manifestations but the complication associated like Dengue hemorrhagic fever and Dengue shock syndrome are more common in DEN-2 and 3 serotypes. The main Patho-physiological mechanisms involved in development of complications include endothelial damage and increased permeability, leading to plasma leakage into extra vascular spaces and serous effusions with high protein content. These effusions are collected in the serous cavities causing pleural, pericardial effusions and ascites. Collections of this leakage also produce an edema of gall bladder wall and gastric mucosa leading to Dyspepsia syndrome. These collections are formed mostly by the $5^{\text {th }}$ or $7^{\text {th }}$ day of the disease. Changing scenario of clinical manifestations and the pattern and prevalence of different serotypes make the type of clinical presentations and timing of development of radiological findings uncertain or unspecific for a clear diagnosis during an early time of outbreak. ${ }^{7}$

Serological tests are still the mainstay in diagnosis of Dengue fever and to differentiate from other causes of febrile illness like typhoid, malaria and spotted fevers. However availability of rapid diagnostic tests by determining presence of NS1 Antigen can help in early diagnosis of dengue fever. But additional diagnostic modalities like ultra sonogram of abdomen; chest can be sought for early diagnosis before development of complications. The early findings which have been quoted in various literatures include combination of findings like thickness of gall bladder wall, pleural, pericardial effusion and ascites. However the degree of accuracy is quite variable in different studies. Hence we evaluated the Ultrasonographic findings during an epidemic and its correlation with platelet counts and different age groups.

In our study, the incidence of males was higher than females and the common age of occurrence was 11-29 years as reported by many studies globally. However some of the studies reported a higher incidence of dengue fever during the $4^{\text {th }}$ and $5^{\text {th }}$ decade of life. ${ }^{8}$ Among the Ultrasonographic findings reported, thickness of the gall bladder wall was the commonest finding in our study with $65.08 \%$ followed nearby with Ascites with $62.70 \%$ and pleural effusion (49.21\%). Findings of our study were in par with the reports of Joshi $\mathrm{P}$ who reported an incidence of $72 \%$ of gall bladder wall thickness in his study and pleural effusion with $64 \%$ in his study. ${ }^{9}$ However our findings were in contrast to the findings of Setiawan MW et al who reported ascites as most common Ultrasonographic finding (76\%) in cases of dengue fever followed by pleural effusion (68\%) in their study. This can be explained by the reason that the study group in their study comprised mostly of paediatric age group. ${ }^{10}$ Spleenomegaly and Hepatomegaly were seen in $30.16 \%$ and $28.57 \%$ of cases in our study. 82 cases in our study (21.69\%) revealed no abnormal findings on ultra sonogram and with 39 cases $(43.82 \%)$ with a platelet count between 80,000 -1, $50,000,25$ cases with platelet count of $>1,50,000$ and 18 cases with platelet count of 40,000 -80,000. Most of the cases with thrombocytopenia had evidences of plasma leakage on ultra sonogram with thickness of gall bladder wall, pleural effusion and ascites which may suggest an early evaluation of ultra sonogram to find out cases progressing towards Dengue hemorrhagic fever. Most of the studies reveal that some kind of findings will be observed in all cases of severe thrombocytopenia $(<40,000)$. In our study, thickness of the gall bladder wall was the most sensitive finding and pleural effusion was the most specific finding. Findings of Motla M et al also revealed, gall bladder wall thickness was the most sensitive finding but ascites as the specific finding which is contrary to the finding of our study. ${ }^{11}$ Most of the studies were in concordance with the findings of our study with thickening of gall bladder as the most common finding in their studies. ${ }^{12}$ Studies done by Asghar J et al demonstrated ascites as the most common finding in his study but the reasons for this are multifactorial regarding difference in age group, during this study the clinical scenario of the most of the patients developed significant thrombocytopenia after 5 th day of febrile illness and the Ultrasonographic finding $\mathrm{s}$ were recorded before development of thrombocytopenia in few cases which may be the reason GB wall thickening as the most common finding in our study. ${ }^{13}$

Hepatomegaly and spleenomegaly were the least common findings observed in our study in all the age groups except in Group -1 where hepatomegaly was more than the cases of Pleural effusion which is contrary to the findings of Santosh $\mathrm{VR}$ et al who reported ascites as the least common finding 
in his study. But most of the studies universally reported organomegaly as the least common finding. The reason may be spleenomegaly is observed mostly in cases of Dengue Hemorrhagic fever and shock syndrome most commonly. In our study the incidence of DHF was only $4 \%$ where ascites and effusions were more common than organomegaly. ${ }^{14}$ In our study, with correlation to combination of USG findings, most common combination was Thickening of the gall bladder wall with associated pleural effusion and followed by hepatomegaly and organomegaly. Majority of the cases with GBT and Pleural effusion had platelet counts in range of 40000-80,000 whereas cases with HPM and Spleenomegaly had platelets $80,000-1,50,000$ and more than $1,50,000$. This clearly explains that sever to moderate thrombocytopenia is associated with development of plasma leakage and collection of fluids in the serous cavities. A combination of all findings excluding hepatomegaly was also seen in adequate cases with severe to moderate thrombocytopenia. This type of study with combination of USG findings with platelet counts were not done and is first of its type reported.

Limitation of the study was as the study was done in a tertiary care hospital, majority of the cases were referred cases after diagnosis and duration of febrile illness was more than $5^{\text {th }}$ or $7^{\text {th }}$ day. The follow up findings of USG were not done to evaluate the accuracy and time of resolution of the findings. To conclude, from our study Gall bladder wall thickening was the most sensitive and pleural effusion was the most specific finding in our study. Gall bladder wall thickening, pleural effusion and ascites were most commonly seen in patients with severe to moderate thrombocytopenia. Gall bladder wall thickening and pleural effusion was the commonest combination seen in most of cases with severe thrombocytopenia. USG is a relevant and important tool for early detection of plasma leakage signs and helps in follow up of patients who have a risk to progress to DHF. GB thickening with or without polyserosistis in a febrile patient should suggest the possibility of Dengue fever during an epidemic outbreak.

\section{REFERENCES}

1. World Health Organisation. Dengue haemorrhagic fever; diagnosis, treatment, prevention, and control. Geneva: WHO; 1997.

2. WHO. Health Situation in South East Asian Region 2001-2007.New Delhi: Regional Office for SEAR; 2008.

3. WHO. Dengue and Dengue Haemorrhagic Fever. Factsheet No 117, Revised May, 2008. Geneva: World Health Organization; 2008. Available from: http:// www.who.int/mediacentre/factsheets/fs117/en/

4. New Delhi: Regional Office for SEAR; 2008. WHO. Health Situation in South East Asian Region 20012007.

5. New Delhi: Ministry of Health and Family Welfare; 2006. Internet, Government of India. National Vector Bourne Disease Control Programme.

6. Jawetz, Melwick . 24th ed. McGraw Hill, Lange Publications; 2007. Adelberg's Medical Microbiology; pp. 350-55.

7. Leitmeyer KC, Vaughn DW, Watts DM, Salas R,
Villalobos I, de Chacon,et al. Dengue virus structural differences that correlate with pathogenesis. J Virol 1999;73(2): 4738-47.

8. Potts JA, Rothman AL. Clinical and laboratory features that distinguish dengue from other febrile illnesses in endemic populations. Trop Med Int Health 2008; 13 (2):1328-40.

9. Joshi P, Rathnam VG, Sharma S. USG findings in dengue haemorrhagic fever - our experience in the recent epidemic. Ind J Radiol Imag 1997;7 (4): 189-92.

10. Setiawan MW, Samsi TK, Pool TN, Sugianto D, Wulur H. Gallbladder wall thickening in dengue hemorrhagic fever: An ultrasonographic study. J Clin Ultrasound. 1995; 23 (6): 357-62.

11. Motla M, Manaktala S, Gupta V, Aggarwal M, Bhoi SK, Aggarwal P, et al. Sonographic evidence of ascites, pleura-pericardial effusion, and gall bladder wall edema as non-invasive, rapid diagnostic markers for dengue fever. Prehosp Disaster Med.2011;26 (5);335-41.

12. Vedaraju KS, Kumar KRV, Vijayaraghavachari TV. Role of Ultrasound in the Assessment of Dengue Fever. Int J Sci Stud 2016;3(10):59-62.

13. Asghar J, Farooq K. Radiological appearance and their significance in the management of dengue hemorrhagic fever. Pak J Med Health Sci. 2011;5 (3): 685-92.

14. Santhosh VR, Patil PG, Srinath MG, Kumar A, Jain A, Archana M.Sonography in the diagnosis and assessment of dengue Fever. J Clin Imaging Sci 2014;4 (1):14.

Source of Support: Nil; Conflict of Interest: None

Submitted: 05-05-2018; Accepted: 09-06-2018; Published online: 15-06-2018 